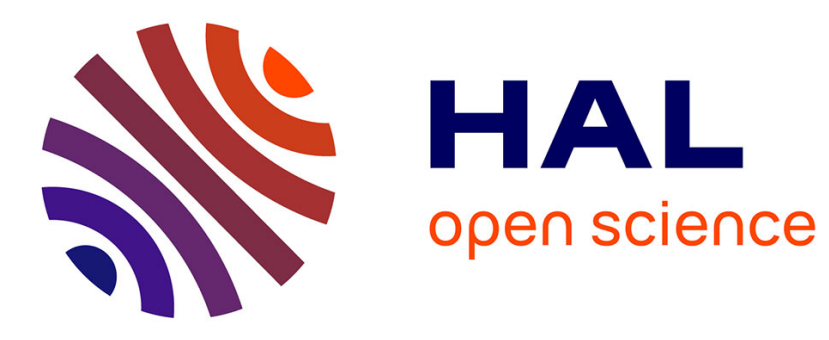

\title{
Homophase signals separation for Volterra series identification
}

Damien Bouvier, Thomas Hélie, David Roze

\section{To cite this version:}

Damien Bouvier, Thomas Hélie, David Roze. Homophase signals separation for Volterra series identification. 57th IEEE Conference on Decision and Control (CDC 2018), Dec 2018, Miami Beach, FL, United States. hal-01930662

\section{HAL Id: hal-01930662 \\ https://hal.science/hal-01930662}

Submitted on 22 Nov 2018

HAL is a multi-disciplinary open access archive for the deposit and dissemination of scientific research documents, whether they are published or not. The documents may come from teaching and research institutions in France or abroad, or from public or private research centers.
L'archive ouverte pluridisciplinaire HAL, est destinée au dépôt et à la diffusion de documents scientifiques de niveau recherche, publiés ou non, émanant des établissements d'enseignement et de recherche français ou étrangers, des laboratoires publics ou privés. 


\title{
Homophase signals separation for Volterra series identification
}

\author{
Damien Bouvier ${ }^{1}$, Thomas Hélie ${ }^{1}$ and David Roze ${ }^{1}$
}

\begin{abstract}
This article addresses the identification of nonlinear systems represented by Volterra series. To improve the robustness of state-of-the-art estimation methods, we introduce the notion of "homophase signals", for which a separation method is given. Those homophase signals are then used to derive a robust identification process. This prior step is similar to nonlinear homogeneous order separation, in which amplitude relations are used to separate the orders of a Volterra series, but offers a better conditioning by using phase deviations rather than amplitudes.

First an academic phase-based method using complex-valued test signals is introduced for separating nonlinear orders. Second this notion of phase deviation is extended to real-valued signals, which leads to the design of the proposed homophase signals separation method. Finally, a new identification process is derived using the homophase signals.

Simulations are used to highlight the benefits of the proposed identification process in comparison to the standard approach.
\end{abstract}

\section{INTRODUCTION}

This paper introduces a new nonlinear homophase separation method using phase as a discriminant factor for improving Volterra series identification. Contrary to linear systems and filters, the topic of nonlinear system identification still causes serious difficulties. One approach, based on series expansion (like Volterra series), has begun in the 40's with the seminal work of Wiener [1]. Since then, many methods have been developed.

Cross-correlation methods [2] take advantage of the orthogonality property of Wiener series (a probabilistic version of the Volterra series); they were further improved in [3], and developed in frequency domain in [4]. Timedomain probing methods rely on the use of impulse-dirac for estimating Volterra kernels [5], [6]. Their frequency domain counterparts were also developed [7]-[9]. Volterra kernels identification problem can also be expressed as a linear system, resulting in the use of Least-Squares estimation methods. Solving can then be made in several ways, e.g. using an orthogonalization on the measured data [10] or a stochastic approximation algorithm [11]. A thorough and up-to-date overview of works about Volterra series and Volterra kernel identification can be found in [12].

Depending on the kind of method used, Volterra series identification can be improved in several ways. Tensor decomposition [13] or projections on orthogonal basis (Laguerre [14], Kautz [15] or Generalized Orthogonal basis [16]) greatly reduces the number of parameters to

\footnotetext{
${ }^{1}$ All authors are with the Team Sound Signals and Systems : Audio/Acoustics, instruMents (S3AM), UMR 9912 IRCAM-CNRS-UPMC, 1, Place Igor Stravinsky, 75004, France. Correspondence should be send to damien.bouviereircam. fr
}

estimate, and so, the overall complexity. Besides, a system can be approximated using a block structure approach (Hammerstein, Wiener, Wiener-Hammerstein, etc), for which there exist specific estimation methods [17]-[19].

In this work, we choose to improve identification by using a prior separation step. This is generally processed by separating nonlinear homogeneous contributions using a collection of amplitude gains applied to a common input signal [7]. But this method has limitations (poor-conditioning, weak robustness to noise measurement). To improve robustness, we introduce the concept of homophase signal contribution, and propose a new method to separate homogeneous phase contributions using only phase deviations between signals. Finally, a new identification process based on those homophase signals is derived, and presented in a Least-Squares framework. Part of the results were already presented in a previous conference paper [20]; novelties of this paper includes thorough robustness analysis of the presented separation methods and the new identification process for truncated Volterra series. All presented methods and algorithms are implemented in an open-source Python toolbox ${ }^{1}$.

This paper is organized as follows: in Section II, we give some mathematical foundations for the Volterra series paradigm and announce the problem statement. Section III proposes a new method of separation for complex-valued input-output signals, solely based on phase. In Section IV, the concept of homophase signal is introduced, and the main result of the paper is presented. This is used in Section V to derive a new Volterra series identification method. Finally, evaluation and comparison are made in Section VI, before giving some conclusions and perspectives.

\section{VOLTERRA SERIES AND PROBLEM STATEMENT}

\section{A. Volterra series}

This section presents an overview of the Volterra formalism; more details can be found in e.g. [21], [22].

Definition 1 (Volterra series): A nonlinear causal timeinvariant system is described by a Volterra series $\left\{h_{n}\right\}_{n \in \mathbb{N}^{*}}$ if, for all input signals $u$ such that $\|u\|_{\infty}<\rho$, the output signal $y$ is given by the following Volterra operator $V$

$$
y=V[u]=\sum_{n=1}^{\infty} V_{n}[u, \ldots, u],
$$

\footnotetext{
${ }^{1}$ Available at https://github.com/d-bouvier/pyvi.
} 
where $V_{n}$ are the Volterra operators of order $n$, given for all time $t$ by

$V_{n}\left[u_{1}, \ldots, u_{n}\right](t)=\int_{\mathbb{R}_{+}^{n}} h_{n}\left(\tau_{1}, \ldots, \tau_{n}\right) \prod_{i=1}^{n} u_{i}\left(t-\tau_{i}\right) \mathrm{d} \tau_{i}$,

and with $\rho$ the convergence radius of the power series $\sum_{n=1}^{+\infty}\left\|h_{n}\right\|_{1} x^{n}$. Functions $h_{n}$ are called Volterra kernels of the system. Signals $y_{n}=V_{n}[u, \ldots, u]$ are called nonlinear homogeneous order contributions (or nonlinear orders for short).

The Volterra formalism can be seen as an extension of the linear convolution (used for representation of time-domain filters) on a Taylor-like series expansion. It can only represent dynamical systems whose nonlinearities are analytic, and cannot describe phenomena like hysteresis, sub-harmonics or chaotic behavior. Nonetheless it has been shown in [23] that a Volterra series operator can be used to approximate any fading-memory time-invariant continuous nonlinear operator.

Remark 1 (Non-unicity of kernels): It can easily be seen from (2) that, for a given system, kernels are not uniquely defined (any permutation of the $\tau_{i}$ 's let $y_{n}$ invariant). Uniquelydefined kernels can be specified, such as the symmetric kernel (which is invariant to any permutation of its arguments) or triangular kernel [21].

Property 1 (Properties of the operator $V_{n}$ ): Operator $V_{n}$ is multilinear, i.e. for any signals $u_{1}, \ldots, u_{n}$ and $v$, and any scalars $\lambda, \mu$,

$$
\begin{aligned}
& V_{n}\left[u_{1}, \ldots, \lambda u_{k}+\mu v, u_{k+1}, \ldots, u_{n}\right]= \\
& \quad \lambda V_{n}\left[u_{1}, \ldots, u_{n}\right]+\mu V_{n}\left[u_{1}, \ldots, v, u_{k+1}, \ldots, u_{n}\right]
\end{aligned}
$$

This implies that $V_{n}$ is a homogeneous operator of degree $n$, i.e. for any signals $u_{1}, \ldots, u_{n}$ and scalar $\alpha$,

$$
V_{n}\left[\alpha u_{1}, \ldots, \alpha u_{n}\right]=\alpha^{n} V_{n}\left[u_{1}, \ldots, u_{n}\right] .
$$

In the sequel, truncated Volterra series are used, where $N$ denotes the truncation order of the system. Furthermore, convergence conditions are always assumed to be met, and symmetry of kernels $h_{n}$ (and therefore of operators $V_{n}$ ) is supposed.

\section{B. Problem statement}

Most of Volterra identification methods exploit directly the raw output signal $y$ in order to identify a set of kernels $\left\{h_{n}\right\}, n=1, \ldots, N$ (as shown in Figure 1a). One important difficulty of this simultaneous estimation lies in the relative differences between amplitudes of the homogeneous orders $y_{n}$; indeed, for a system that allows a Volterra series representation, amplitudes generally decrease as the order $n$ increases, thus making estimation of the higher-order kernels less reliable.

In order to circumvent this difficulty, it is possible to divide the identification into 2 steps (as shown in Figure 1b):

1) Separate the nonlinear homogeneous orders $y_{n}, n=$ $1, \ldots, N$ from a set of output signals;

2) Identify separately the kernels $h_{n}$ from each signal $y_{n}$.

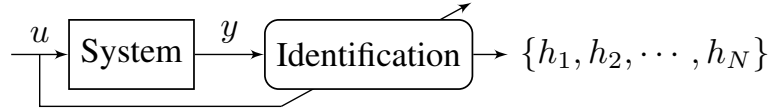

(a) Direct identification

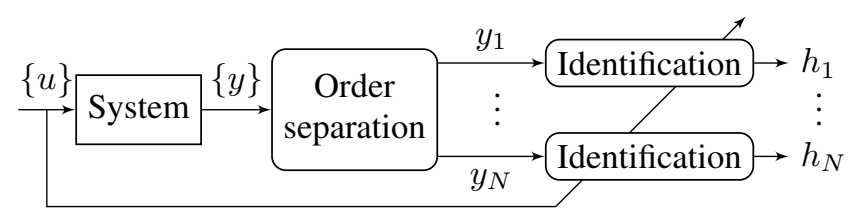

(b) Identification on separated order

Fig. 1. Identification process without (a) and with (b) prior order separation.

This two-step approach increases the efficiency and robustness of the overall kernels estimation by splitting the identification process into $N$ smaller problems. Furthermore, this procedure is modular, meaning that any existing identification methods can be used in step 2 .

However, the only available order separation method is limited to the first few orders in practice [7], [24]. This method is shortly recalled below.

Let $u$ be a signal, and $\alpha_{k} \in \mathbb{R}, k=1, \ldots, K$ a set of amplitude gains. Denote $u_{k}(t)=\alpha_{k} u(t)$ a collection of $K$ tests signals, and $z_{k}(t)=V\left[u_{k}\right](t)$ the corresponding outputs through the system. From homogeneity property (4), we can write the following linear system, $\forall t$,

$$
\begin{gathered}
{\left[\begin{array}{c}
z_{1} \\
z_{2} \\
\vdots \\
z_{K}
\end{array}\right](t)=\left[\begin{array}{cccc}
\alpha_{1} & \alpha_{1}^{2} & \ldots & \alpha_{1}^{N} \\
\alpha_{2} & \alpha_{2}^{2} & \ldots & \alpha_{2}^{N} \\
\vdots & \vdots & \ddots & \vdots \\
\alpha_{K} & \alpha_{K}^{2} & \ldots & \alpha_{K}^{N}
\end{array}\right] \cdot\left[\begin{array}{c}
y_{1} \\
y_{2} \\
\vdots \\
y_{N}
\end{array}\right](t)} \\
\boldsymbol{Z}(t)= \\
\boldsymbol{A}
\end{gathered}
$$

where $y_{n}$ are the homogeneous orders of the system in response to the input signal $u$. Since $\boldsymbol{A}$ is a Vandermonde matrix, it is (pseudo-)invertible if and only if $\alpha_{k}$ are all different from zero and each other, and the number of test signals $K$ is greater or equal to the truncation order $N$. In this case, it is possible to recover orders $y_{n}$. In the following, this method will be referred as the Amplitude Separation (AS) method. It has been used for identification purposes in e.g. [7], [25], [26].

The use of amplitudes $\alpha_{k}$ yields several limitations:

- for high amplitudes, the system can saturate, or be excited outside the validity domain (of the series or of its truncated version);

- for small amplitudes, high orders can be hidden in measurement noise.

Another drawback is that the real-valued Vandermonde matrix to invert is known to be rapidly ill-conditioned as its size grows. Even if it is possible to circumvent this by using Newton Recursive or Lagrange Recursive method [27, Algorithm 4.6.1 and 4.6.2], or by using more than $N$ test signals, the use of the AS method is limited to the first few orders in practice. The main idea of this paper consists of exploiting phase deviations rather than amplitude differences 


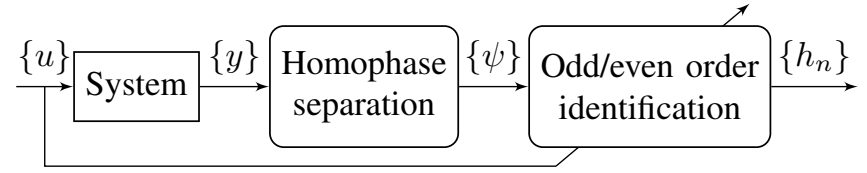

Fig. 2. Identification process with prior homophase signal separation.

as separating factors. This leads to a new expression of the output signal of a Volterra series as the sum of homophase contributions, for which a separation method is designed. From those homophase signals a new identification process is proposed, where odd and even kernels are separately estimated from odd and even homophase signals (see Fig. 2).

\section{Phase-BASEd ORder SeParation Method FOR COMPLEX-VALUED SIGNALS}

This section handles the theoretical case of complexvalued input and output signals. It allows the examination of phase exploitation in the design of a robust separation method.

\section{A. Method presentation}

Let $u$ be a complex-valued signal and $w=e^{j 2 \pi / N}$ be the first $N^{\text {th }}$ unit-root. Denote $u_{k}(t)=w^{k-1} u(t), k=1, \ldots, N$ a collection of $N$ test signals, and $z_{k}(t)=V\left[u_{k}\right](t)$ the corresponding outputs through the system. From homogeneity property (4), it comes

$$
V\left[w^{k-1} u\right](t)=\sum_{n=1}^{N} w^{n(k-1)} y_{n}(t),
$$

where $y_{n}$ are the homogeneous orders of the system in response to the input signal $u$. It follows that, $\forall t$,

$$
\begin{aligned}
& {\left[\begin{array}{c}
z_{1} \\
z_{2} \\
\vdots \\
z_{N}
\end{array}\right](t)=\left[\begin{array}{cccc}
1 & 1 & \ldots & 1 \\
w & w^{2} & \ldots & 1 \\
\vdots & \vdots & \vdots & \\
w^{N-1} & w^{2 N-2} & \ldots & 1
\end{array}\right] \cdot\left[\begin{array}{c}
y_{1} \\
y_{2} \\
\vdots \\
y_{N}
\end{array}\right](t)} \\
& \boldsymbol{Z}(t)=\boldsymbol{W}_{\boldsymbol{N}} \quad \cdot \boldsymbol{Y}(t),
\end{aligned}
$$

where $W_{N}$ is, within column permutation, the Discrete Fourier Transform (DFT) matrix ${ }^{2}$ of order $N$. It is important to note that the DFT does not apply on time, but on the homogeneous nonlinear orders. A new separation method is obtained by inverting (7), which is more robust than inverting (5) since $\boldsymbol{W}_{N}$ is unitary. This method will be referred as the Complex Phase Separation (CPS) method.

Remark 2: In the linear algebra formalism, AS and CPS methods look very similar. But they differ in their functioning; AS relies on the amplitude difference between signals whereas CPS uses phase deconstruction and reconstruction to recover the nonlinear orders. This difference can also be viewed graphically on the complex plane (see Fig. 3); in CPS, all the factors $w^{k}$ and their power will be on the unit

\footnotetext{
${ }^{2}$ It suffices to consider vectors $\widehat{\boldsymbol{Y}}=\left[y_{N}, y_{1}, \ldots, y_{N-1}\right]^{T}$ to recover the usual DFT matrix.
}

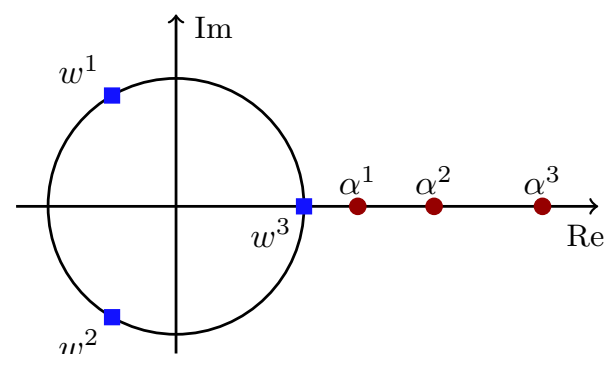

Fig. 3. Graphical comparison of the evolution of the separating factors for the classic AS method ( $\alpha \in \mathbb{R}$, red circle) and proposed CPS method $\left(w=e^{j 2 \pi / N}\right.$, blue square) through a Volterra series truncated to order $N=3$.

circle, and can only be on one of the $N$ unit-roots; in AS, factors $\alpha_{k}$ and their powers lie on the real axis, and converge towards 0 (if $\left|\alpha_{k}\right|<1$ ) or diverge (if $\left|\alpha_{k}\right|>1$ ) as the truncation order increases.

Obviously, because the test signals $w^{k-1} u(t)$ are complexvalued, CPS method is not applicable in practice. This is why the idea of using phase deviations is extended to real-valued signals in Section IV.

\section{B. Properties of the CPS method}

The CPS method comes with interesting properties concerning robustness to noise and truncation error:

1) Noise reduction: Suppose that each measured output $z_{k}$ is perturbed by an independent and identically distributed (i.i.d.) Gaussian noise following a circular symmetric complex normal distribution of variance $\sigma^{2}$ (see [28] for theory on complex-valued normal distributions). Then relation (7) becomes, $\forall t$,

$$
\widetilde{\boldsymbol{Z}}(t)=\boldsymbol{W}_{\boldsymbol{N}} \boldsymbol{Y}(t)+\varepsilon(t)
$$

where $\varepsilon$ is a multivariate i.i.d. circular symmetric complex Gaussian noise of covariance matrix $\boldsymbol{\Sigma}=\sigma^{2} \mathcal{I}$, with $\mathcal{I}$ the identity matrix. The best estimator $\widetilde{\boldsymbol{Y}}$ of $\boldsymbol{Y}$ in a least meansquare sense is computed by

$$
\begin{aligned}
\widetilde{\boldsymbol{Y}}(t) & =\boldsymbol{W}_{\boldsymbol{N}}^{-1} \widetilde{\boldsymbol{Z}}(t) \\
& =\boldsymbol{Y}(t)+\widetilde{\boldsymbol{\varepsilon}}(t) .
\end{aligned}
$$

The new noise vector $\widetilde{\varepsilon}$ is also a multivariate i.i.d. circular symmetric complex Gaussian noise of covariance matrix $\widetilde{\Sigma}$ given by

$$
\begin{aligned}
\widetilde{\boldsymbol{\Sigma}} & =\boldsymbol{W}_{\boldsymbol{N}}^{-1} \boldsymbol{\Sigma}\left(\boldsymbol{W}_{\boldsymbol{N}}^{-1}\right)^{*} \\
& =\frac{\sigma^{2}}{N} \mathcal{I} .
\end{aligned}
$$

Equation (10) shows that using CPS method, Signal-to-Noise Ratio (SNR) between terms $y_{n}$ and error $\widetilde{\varepsilon}$ due to noise measurement is improved by a factor of $\sqrt{N}$. In comparison, noise sensitivity of AS method greatly depends on the chosen factors $\alpha_{k}$, and is different for each order $n$. 
2) Nonlinear order aliasing and rejection factor: Given the $N$-periodicity of any $N^{\text {th }}$ root of unity $w$, the output of a Volterra series in response to an input $w u(t)$ is

$$
V[w u](t)=\sum_{n=1}^{N} w^{n} \sum_{r=0}^{\infty} y_{n+r N}(t),
$$

where $y_{n}$ are the homogeneous orders of the system in response to the input signal $u$. Thus, by applying CPS method of order $N$ to an infinite Volterra series, estimation of nonlinear orders $\widetilde{y}_{n}, n=1, \ldots, N$ yields:

$$
\widetilde{y}_{n}=y_{n}+\sum_{r=1}^{\infty} y_{n+r N} .
$$

Equation (12) reveals that estimation $\widetilde{y}_{n}$ is perturbed by a residual term $\sum_{r=1}^{\infty} y_{n+r N}$, which is structured as a nonlinear order aliasing.

This aliasing artifact permits to create higher-order rejection by using amplitude as a contrast factor between the wanted terms $y_{n}$ and the perturbation signals $y_{n+r N}$. Taking inputs $u_{k}=\rho w^{k} u$, where $0<\rho<1$, and using CPS yields

$$
\widetilde{y}_{n}=\rho^{n}\left(y_{n}+\sum_{r=1} \rho^{r N} y_{n+r N}\right) \text {. }
$$

This creates a ratio $1 / \rho^{N}$ between the desired signal $y_{n}$ and the first perturbation $y_{n+N}$. Hence parameters $N$ and $\rho$ enables to reach a wanted ratio between signal and higher-order perturbations.

It is important to note that using a contrast factor will impede the SNR amelioration presented in III-B.1. Derivations similar to (8-10), show that the SNR increment for each order $n$ will now be $\rho^{n} \sqrt{N}$, which is inferior to $\sqrt{N}$ for $0<\rho<1$.

Taking into account both order aliasing and noise reduction properties, parameters $N$ and $\rho$ allows to control error estimation due to noise measurement and higher-orders. The overall method is described in Algorithm 1. Increasing the truncation order (and therefore the number of needed test signals) leads to a more robust order separation method (in terms of sensitivity to noise and truncation order). The main drawback is that, in practice, it is not possible to use complex input and output signals on a physical system.

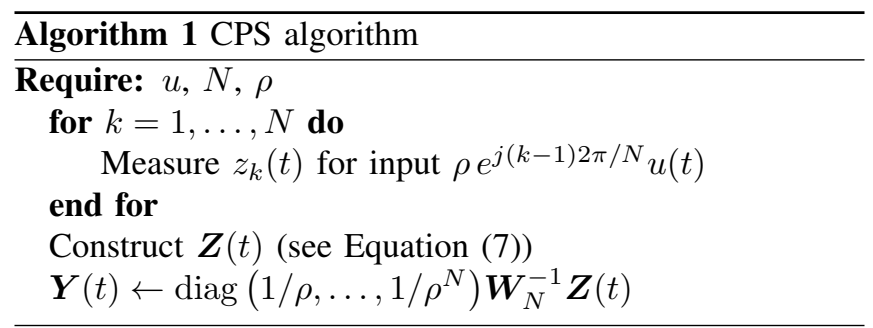

\section{HOMOPHASE SIGNALS SEPARATION METHOD FOR REAL-VALUED SIGNALS}

We now extend the idea of a preprocessing separation step through phase deviations to real-valued signals. This leads to the novel concept of homophase signal contribution, and the design of a robust method to separate them.

\section{A. Method presentation}

We begin by introducing a new notation that will be useful for the derivation of our method.

Definition 2 (Interconjugate term): Let $u$ be a complexvalued signal and $V_{n}$ be a symmetric Volterra operator of order $n$. Then we note $V_{n, q}$ the complex-valued interconjugate term defined by

$$
V_{n, q}(t)=V_{n}[\underbrace{u, \ldots, u}_{n-q \text { times }}, \underbrace{\bar{u}, \ldots, \bar{u}}_{q \text { times }}](t) .
$$

Example 1: Let $u(t)=e^{j 2 \pi f t}$ be a complex sinusoidal signal. Then it can be shown that interconjugate terms $V_{n, q}$ are also sinusoids, of frequency $(n-2 q) f$, with phase and amplitude depending on the system.

Remark 3: Terms $V_{n, 0}$ (respectively $V_{n, n}$ ) are the output of order $n$ of the system excited by the signal $u$ (resp. $\bar{u}$ ).

Property 2: By symmetry of $V_{n}$, it is straightforward to show that $V_{n, q}=\overline{V_{n, n-q}}$, and that for even $n$, term $V_{n, n / 2}$ is real.

Theorem 1: Let $u$ be a complex-valued signal and $\theta$ a complex scalar on the unit circle. Let $y_{n}$ be the order $n$ output of the system in response to the input signal $\operatorname{Re}[\theta u(t)]$. Then

$$
y_{n}(t)=\frac{1}{2^{n}} \sum_{q=0}^{n}\left(\begin{array}{l}
n \\
q
\end{array}\right) \theta^{n-2 q} V_{n, q}(t) .
$$

Proof: Using the multilinearity property (3) and the symmetry of $V_{n}$, it follows that

$$
\begin{aligned}
y_{n}(t) & =V_{n}[\operatorname{Re}[\theta u(t)], \ldots, \operatorname{Re}[\theta u(t)]](t) \\
& =\frac{1}{2^{n}} V_{n}[\theta u+\overline{\theta u}, \ldots, \theta u+\overline{\theta u}](t) \\
& =\frac{1}{2^{n}} \sum_{q=0}^{n}\left(\begin{array}{l}
n \\
q
\end{array}\right) \theta^{n-q} \bar{\theta}^{q} V_{n}[\underbrace{u, \ldots, u}_{n-q \text { times }}, \underbrace{\bar{u}, \ldots, \bar{u}}_{q \text { times }}](t) \\
& =\frac{1}{2^{n}} \sum_{q=0}^{n}\left(\begin{array}{l}
n \\
q
\end{array}\right) \theta^{n-2 q} V_{n, q}(t),
\end{aligned}
$$

which concludes the proof.

This notion of interconjugate terms allows to rewrite the output of a truncated Volterra series, as the following result shows.

Theorem 2: Let $u$ be a complex-valued signal and $\theta$ a complex scalar on the unit circle. Let $y$ be the output of the system excited by $\operatorname{Re}[\theta u(t)]$. Then

$$
y(t)=\sum_{p=-N}^{N} \theta^{p} \psi_{p}(t),
$$


where $\psi_{p}$ are the homophase signals defined by

$$
\psi_{p}(t)=\left\{\begin{array}{c}
\sum_{\substack{n=|p| \\
n \equiv p(\bmod 2)}}^{N} \frac{1}{2^{n}}\left(\begin{array}{c}
n \\
\frac{n-p}{2}
\end{array}\right) V_{n, \frac{n-p}{2}}(t) \quad, p \neq 0 \\
\sum_{\substack{n=2 \\
n \equiv 0(\bmod 2)}}^{N} \frac{1}{2^{n}}\left(\begin{array}{c}
n \\
n / 2
\end{array}\right) V_{n, n / 2}(t) \quad, p=0
\end{array} .\right.
$$

Proof: Using (1) and (15) it follows that

$$
\begin{aligned}
y(t) & =\sum_{n=1}^{N} \frac{1}{2^{n}} \sum_{q=0}^{n}\left(\begin{array}{c}
n \\
q
\end{array}\right) \theta^{n-2 q} V_{n, q}(t) \\
= & \sum_{n=1}^{N} \frac{1}{2^{n}} \sum_{\substack{p=-n \\
p \equiv n(\bmod 2)}}^{n}\left(\begin{array}{c}
n \\
\frac{n-p}{2}
\end{array}\right) \theta^{p} V_{n, \frac{n-p}{2}}(t) \\
= & \sum_{\substack{p=-N \\
p \neq 0}}^{N} \sum_{\substack{n=|p| \\
n \equiv(\bmod 2)}}^{N} \frac{1}{2^{n}}\left(\begin{array}{c}
n \\
\frac{n-p}{2}
\end{array}\right) \theta^{p} V_{n, \frac{n-p}{2}}(t) \\
& +\sum_{\substack{n=2 \\
n \equiv 0(\bmod 2)}}^{N} \frac{1}{2^{n}}\left(\begin{array}{c}
n \\
n / 2
\end{array}\right) V_{n, n / 2}(t) \\
= & \sum_{p=-N}^{N} \theta^{p} \psi_{p}(t),
\end{aligned}
$$

which concludes the proof.

Example 2: Let $u(t)=e^{j 2 \pi f t}$ be a complex sinusoidal signal. Then each homophase signal $\psi_{p}$ is a complex sinusoid of frequency $p f$ built from contributions of orders $n \geq p$.

Property 3: It results from Property 2 that $\psi_{p}=\overline{\psi_{-p}}$.

Using (16), it is straightforward to prove the following result.

Theorem 3: Let $u$ be a complex-valued signal and $w=$ $e^{j 2 \pi / K}$ with $K=2 N+1$. Denote $u_{k}(t)=\operatorname{Re}\left[w^{k-1} u(t)\right]$ a collection of $K$ test signals, and $z_{k}(t)=V\left[u_{k}\right](t)$ the corresponding outputs through the system. Then

$$
\left[\begin{array}{c}
z_{1} \\
z_{2} \\
\vdots \\
z_{K}
\end{array}\right](t)=\boldsymbol{W}_{\boldsymbol{K}}\left[\begin{array}{c}
\psi_{0} \\
\psi_{1} \\
\vdots \\
\psi_{N} \\
\psi_{-N} \\
\vdots \\
\psi_{-1}
\end{array}\right](t)
$$

where $\boldsymbol{W}_{\boldsymbol{K}}$ is the DFT matrix of order $K$.

Equation (18) shows that it is possible to separate homophase signal contribution in a similar fashion as with CPS method, using a carefully constructed set of signals and an inverse DFT. This will be referred as the Homophase Separation (HPS) method.

The main difference with CPS method is that HPS method does not require to excite the system with complex-valued signals. However, HPS method requires more test signals $(2 N+1$ instead of $N)$, and does not completely separate homogeneous orders $y_{n}$, but only homophase contributions. As will be shown in Section V, homophase signals can be used to greatly improve kernel identification.
Remark 4: The CPS method also processes homophase separation. Indeed, for complex-valued signals, the notion of homophase signals and nonlinear homogeneous order contributions are merged; this similarity is lost as soon as signals are real-valued.

\section{B. Properties of the HPS method}

1) Number of phases and noise reduction: As in the CPS method, the use of an inverse DFT reduces the error due to measurement noise by a factor $\sqrt{K}$ (see section III-B.1). Therefore, for a given truncation order $N$, it is interesting to choose a value of $K$ larger than $2 N+1$. Taking more points on the unit circle builds an interpolation on the phase domain. Therefore, by property of the DFT, this leads to zero-padding in the transform domain, i.e. the estimated vector $\widetilde{\boldsymbol{\Psi}}$ of homophase signals becomes

$$
\widetilde{\boldsymbol{\Psi}}=\left[\begin{array}{llllllllll}
\psi_{0} & \psi_{1} & \ldots & \psi_{N} & 0 & \ldots & 0 & \psi_{-N} & \ldots & \psi_{-1}
\end{array}\right]^{T},
$$

with $K-(2 N+1)$ zeros added.

2) Order aliasing: It can easily be seen from the definition of homophase signals (17) that HPS method will not have the same order aliasing property as CPS. Furthermore, even if it is possible to predict in which homophase signal $\psi_{p}$ higher-order interconjugate terms will appear, the use of a rejection factor to diminish them is not viable.

The overall HPS method, that takes into account the phase domain interpolation, is described in Algorithm 2.

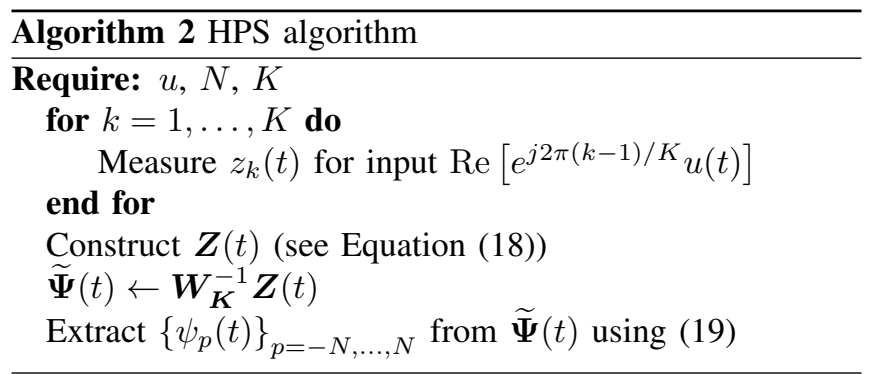

\section{NEW IDENTIFICATION PROCESS BASED ON HOMOPHASE SIGNALS}

This section presents an estimation method for discretetime Volterra kernel. It introduces the identification process for homophase signals in this formalism. The proposed process can be integrated with any identification methods. For sake of simplicity, the estimation part (of Volterra kernels from homophase signals) is chosen as a standard LeastSquare (LS) method.

\section{A. Least-square kernel identification}

The discrete-time version of input-output relation (2) is, at time index $l$,

$V_{n}\left[u_{1}, \ldots, u_{n}\right][l]=\sum_{k_{1}, \ldots, k_{n}=0}^{M-1} h_{n}\left[k_{1}, \ldots, k_{n}\right] \prod_{i=1}^{n} u_{i}\left[l-k_{i}\right]$, 
where $M$ is the kernel memory length (in samples).

Suppose an input signal of length $L$, and denote $\boldsymbol{y}_{\boldsymbol{n}}$ the vector representing discrete signal $y_{n}[l]=V_{n}[u, \ldots, u][l]$, $l=0, \ldots, L-1$. Then, using (20), we write the following matrix relation

$$
y_{n}=\Phi_{n} f_{n}
$$

where $\Phi_{n}$ is the input combinatorial matrix and $f_{n}$ a column vector regrouping all coefficients of $h_{n}$ (see [11] for more details about construction of the combinatorial matrix). Only non-redundant terms of the symmetric form of $h_{n}$ are considered $^{3}$ in $f_{n}$.

Example 3: For an order 2 kernel of memory length $M=$ $3, \Phi_{\mathbf{2}}$ and $\boldsymbol{f}_{\mathbf{2}}$ are given ${ }^{4}$ by

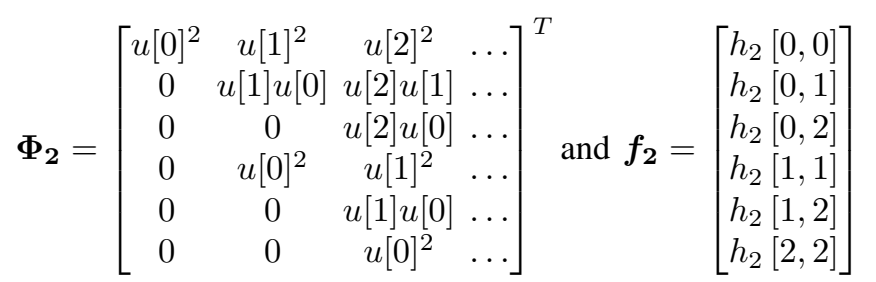

Identifying kernels $h_{n}$ comes down to solving equation (21) for each order $n$. This is possible if $L$ is larger than the number of Volterra coefficients to estimate $\left(\begin{array}{c}M+n-1 \\ n\end{array}\right)$ [13]. This identification process will be referred as order-LS.

If the homogeneous orders $y_{n}$ are not available, but only the direct output $y$, identification is still possible by solving the following relation

$$
y=\Phi f,
$$

with $\boldsymbol{\Phi}=\left[\begin{array}{lll}\boldsymbol{\Phi}_{1} & \ldots & \boldsymbol{\Phi}_{\boldsymbol{N}}\end{array}\right]$ and $\boldsymbol{f}=\left[\begin{array}{lll}\boldsymbol{f}_{1}^{T} & \ldots & \boldsymbol{f}_{\boldsymbol{N}}^{T}\end{array}\right]^{T}$. This identification process will then be referred as direct-LS. In order to improve kernel coefficient estimation, it was proposed in [10] and further developed in [29], [30] to solve (21) or (22) using a QR decomposition of the combinatorial matrix. This is equivalent to applying an orthogonalization procedure onto the basis constructed from of all the nonlinear combinations of the input data (i.e. the columns of $\boldsymbol{\Phi}$ or $\boldsymbol{\Phi}_{n}$ ) and project the output data on this new basis.

\section{B. Kernel identification from homophase signals}

Denote $\boldsymbol{V}_{\boldsymbol{n}, \boldsymbol{q}}$ the vector representing discrete signal $V_{n, q}[l]$, $l=0, \ldots, L-1$. Then, using (20) and the definition of interconjugate term $V_{n, q}$, we write

$$
V_{n, q}=\Phi_{n, q} f_{n}
$$

where $\boldsymbol{\Phi}_{\boldsymbol{n}, \boldsymbol{q}}$ is the input combinatorial matrix corresponding to term $V_{n, q}$. Matrix $\boldsymbol{\Phi}_{n, \boldsymbol{q}}$ is constructed in a similar fashion as $\Phi_{q}$, but conjugated terms have to be taken into account when doing symmetrization ${ }^{5}$.

\footnotetext{
${ }^{3}$ Or equivalently the nonzero terms of the triangular form.

${ }^{4}$ The lexicographical order is used here; this choice has no impact on the estimation process.

${ }^{5}$ For example, combinatorial term $u[l] \overline{u[l-1]}$ and $u[l-1] \overline{u[l]}$ are both linked to the same kernel coefficient $h_{2}[0,1]$, and should thus contribute equally to corresponding term in $\phi_{\mathbf{2}, \mathbf{1}}[l]$
}

Example 4: For an order 2 kernel of memory length $M=$ 3 , combinatorial matrix $\boldsymbol{\Phi}_{\mathbf{2}, \mathbf{1}}$ is given by

$\mathbf{\Phi}_{\mathbf{2}, \mathbf{1}}=\left[\begin{array}{cccc}|u[0]|^{2} & \frac{|u[1]|^{2}}{u[1] \overline{u[0]}+\overline{u[1]} u[0]} & \frac{|u[2]|^{2}}{2} & \ldots \\ 0 & \frac{u[2] \overline{u[1]}+\overline{u[2]} u[1]}{2} & \ldots \\ 0 & 0 & \frac{u[2] \overline{u[0]}+\overline{u[0]} u[2]}{2} & \ldots \\ 0 & |u[0]|^{2} & \frac{|u[1]|^{2}}{2} & \ldots \\ 0 & 0 & \frac{u[1] \overline{u[0]}+\overline{u[1]} u[0]}{2} & \ldots \\ 0 & 0 & \ldots .\left.u[0]\right|^{2} & \ldots\end{array}\right]^{T}$ mophase signals $\psi_{p}, \forall p=-N, \ldots, N$. Combining the homophase signals definition (17) with (23), we obtain the following systems (for odd and even orders respectively):

$$
\begin{aligned}
& {\left[\begin{array}{c}
\psi_{\mathbf{1}} \\
\boldsymbol{\psi}_{\mathbf{3}} \\
\boldsymbol{\psi}_{\mathbf{5}} \\
\vdots
\end{array}\right]=\left[\begin{array}{cccc}
\frac{1}{2} \boldsymbol{\Phi}_{\mathbf{1 , 0}} & \frac{3}{8} \boldsymbol{\Phi}_{\mathbf{3}, \mathbf{1}} & \frac{5}{16} \boldsymbol{\Phi}_{\mathbf{5}, \mathbf{2}} & \ldots \\
\mathbf{0} & \frac{1}{8} \boldsymbol{\Phi}_{\mathbf{3}, \mathbf{0}} & \frac{5}{32} \boldsymbol{\Phi}_{\mathbf{5}, \mathbf{1}} & \ldots \\
\mathbf{0} & \mathbf{0} & \frac{1}{32} \boldsymbol{\Phi}_{\mathbf{5}, \mathbf{0}} & \ldots \\
\vdots & \vdots & \vdots & \ddots
\end{array}\right] \cdot\left[\begin{array}{c}
\boldsymbol{f}_{\mathbf{1}} \\
\boldsymbol{f}_{\mathbf{3}} \\
\boldsymbol{f}_{\mathbf{5}} \\
\vdots
\end{array}\right]} \\
& {\left[\begin{array}{c}
\boldsymbol{\psi}_{\mathbf{0}} \\
\boldsymbol{\psi}_{\mathbf{2}} \\
\boldsymbol{\psi}_{\mathbf{4}} \\
\vdots
\end{array}\right]=\left[\begin{array}{cccc}
\frac{1}{2} \boldsymbol{\Phi}_{2, \mathbf{1}} & \frac{3}{8} \boldsymbol{\Phi}_{4, \mathbf{2}} & \frac{5}{16} \boldsymbol{\Phi}_{\mathbf{6}, \mathbf{3}} & \ldots \\
\frac{1}{2} \boldsymbol{\Phi}_{2, \mathbf{0}} & \frac{1}{4} \boldsymbol{\Phi}_{4, \mathbf{1}} & \frac{15}{64} \boldsymbol{\Phi}_{\mathbf{6}, \mathbf{2}} & \ldots \\
\mathbf{0} & \frac{1}{16} \boldsymbol{\Phi}_{\mathbf{4 , 0}} & \frac{3}{32} \boldsymbol{\Phi}_{\mathbf{6}, \mathbf{1}} & \ldots \\
\vdots & \vdots & \vdots & \ddots
\end{array}\right] \cdot\left[\begin{array}{c}
f_{\mathbf{2}} \\
\boldsymbol{f}_{\mathbf{4}} \\
\boldsymbol{f}_{\mathbf{6}} \\
\vdots
\end{array}\right]}
\end{aligned}
$$

where $\mathbf{0}$ represents null matrices of the appropriate sizes. Kernels can hence be estimated from homophase signals by solving (24) and (25). This identification process will be referred as phase-LS.

Due to the triangular nature of the matrix to invert, it is possible to do the inversion using a technique similar to a back substitution; this method will be referred as iter- $L S$, and is described in Algorithm 3. But, because this approach iterates from higher orders to lower orders, the estimation error is accumulated from order $N$ to 1 . This can be very harmful if the higher homophase signals are hidden in noise, or if the truncation order is underestimated.

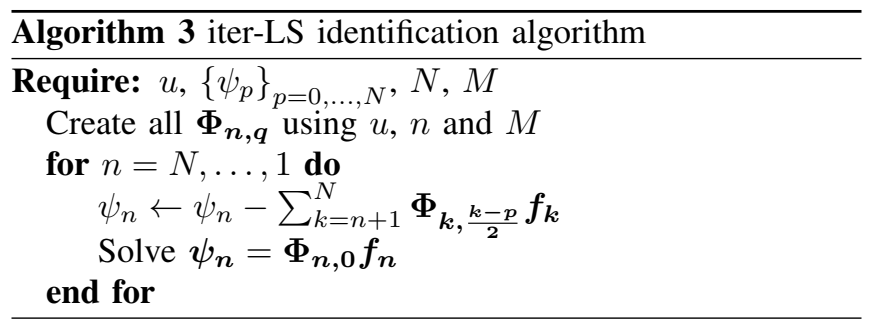

\section{KERNEL IDENTIFICATION EVALUATION ON A SIMULATED SYSTEM}

In this section, numerical experiments are proposed to evaluate the contribution of the separation processes to kernel identification. As order separation do not depend on hypothesis about kernel length or form, a simple academic model with short memory $M$ is used. Usually, true systems exhibit longer response time, and larger $M$ should be used in practice. This leads to difficulties in the identification step, due to the rising number of parameters to estimate. This issue is not addressed here. 


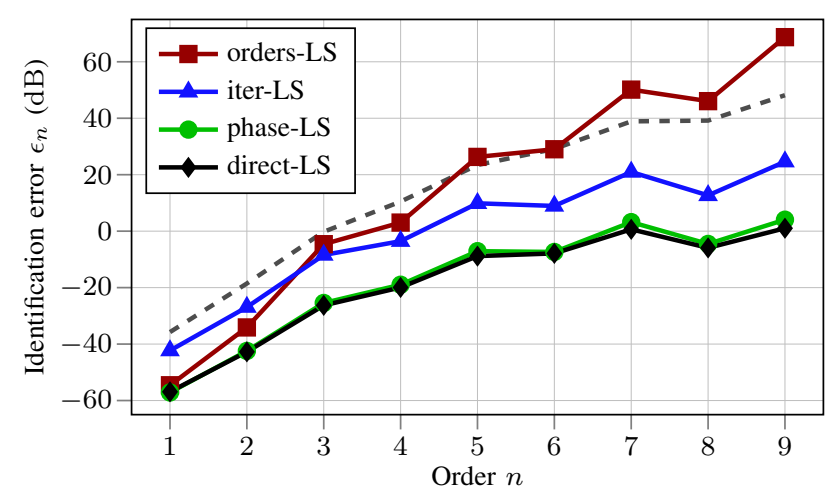

Fig. 4. Comparison of identification error $\epsilon_{n}$ (in dB) w.r.t. order $n$ for the case $K=K_{1}=19$; given results are the median values over all experiments; the dashed line represents the results obtained using direct-LS with a signal of length $L$, given as a reference.

\section{A. Experiment description}

The simulated system is a Volterra series truncated to order 9 , with a finite memory length of $M=5$ samples. All kernels are given by the following formula:

$$
h_{n}\left[k_{1}, \ldots, k_{n}\right]=10^{1-n}(-1)^{\sum_{i} k_{i}} e^{-0.1 \max k} .
$$

Input signals are white Gaussian process of zero-mean and unit variance, with a length of $L=5000$ samples. A perturbation noise (also white and Gaussian) is added to the measured output, so that the overall SNR is $40 \mathrm{~dB}$. Two error measures are used:

- the identification error between true and estimated kernel, given by $\epsilon_{n}=\operatorname{RMS}\left(\widetilde{h_{n}}-h_{n}\right) / \operatorname{RMS}\left(h_{n}\right)$;

- the simulation error between true and reconstructed output signal, given by $\zeta_{n}=\operatorname{RMS}(\widetilde{y}-y) / \operatorname{RMS}(y)$; it is computed for a distinct validation signal (also white and Gaussian with zero-mean and unit variance).

The four presented identification methods are tested and compared: direct-LS, order-LS using prior AS method, iterLS and phase-LS using prior HPS method. As the efficiency of separation methods is impacted by the number of test signals $K$, two experiments are done using (a) $K=K_{1}=19$ (minimum number of test signals required by HPS method) and (b) $K=K_{2}=190$ for both AS and HPS. Furthermore, to make the comparison meaningful, direct-LS method uses the same amount of data points, that is it relies on a test signal of length $K L$. For each test, 10 realizations of the input signal are done.

Gains for AS method are set as $\alpha_{k}=(-1)^{k} \alpha^{\lfloor k / 2\rfloor}$, where value $\alpha$ was optimized to minimize condition number. The used values are 0.8469 for case (a) and 0.9554 for case (b).

\section{B. Results}

Fig. 4 shows the identification error of all methods for case (a). The dashed line represents the results obtained using direct-LS with a signal of length $L$. This quick experiment (only one short measurement is needed) gives poor estimation of kernels for order higher than 3. This is due to the fact that higher order contributions are hidden in noise. The orderLS method gives good results for lower orders: the error on the linear term is sensibly equivalent to the SNR of the measured output. But quality quickly decreases w.r.t. $n$, due to the bad conditioning of the AS method: errors over $0 \mathrm{~dB}$ are obtained for orders higher than 4 . Both methods using the proposed homophase signal separation method (phase-LS and iter-LS) give better results than the one based on AS. The overall better results of phase-LS method over iter-LS is explained by two phenomenas: for higher-orders, phaseLS uses more data to do the estimation than iter-LS (all interconjugate terms $V_{n, q}$ instead of only $\left.V_{n, 0}\right)$; for lowerorders, the iterative process of iter-LS makes the estimation error propagates from order $N$ to order 1. Method direct-LS and phase-LS have similar results over all orders.

Fig. 5 shows the simulation error of all methods w.r.t. their computation time ${ }^{6}$ for both cases (a) and (b). Simulation error follow the trend observed for the kernels identification error: method orders-LS performs poorly and phaseLS outperforms iter-LS of around $20 \mathrm{~dB}$. An improvement of $10 \mathrm{~dB}$ is observed for orders-LS, iter-LS and phase-LS between cases (a) and (b). For iter-LS and phase-LS, this is coherent with the noise reduction property of HPS method (see Section IV-B.1). Furthermore, direct-LS is slightly better than phase-LS in case (a), but the improvement increases up to $15 \mathrm{~dB}$ in case (b). The improvement go along with a much higher computation cost, going from almost $2 \mathrm{~min}$ to more than $20 \mathrm{~min}$. This is due to the augmentation of the number of rows in matrix $\boldsymbol{\Phi}$ for direct-LS, which does not affect methods using prior separation ${ }^{7}$. For those methods, the computation time of the identification step is unchanged, and the duration of the separation step is negligible ${ }^{8}$.

In summary, the proposed phase-LS method gives results close to the direct approach for an equivalent number of data (see Fig. 4). In the direct approach, increasing the measurement length improves identification but also drastically increases computation time. Using prior separation, the number of test signals $K$ can be increased to improve kernel estimation with a negligible impact on computation time (see Fig. 5).

\section{CONCLUSIONS AND PERSPECTIVE}

In this paper, the exploitation of phase deviation in Volterra series output for identification issues has been studied. The concept of homophase signal contribution has been introduced, for which a new homophase separation method has been proposed. Furthermore, a new kernel identification process using those homophase signals has been designed. The robustness property of the homophase separation have been highlighted and explained by optimal condition number of the Discrete Fourier Matrix. The identification built on homophase signals provides results very close to the optimal Least-Squares solution and significantly improves memory

\footnotetext{
${ }^{6}$ Experiments were done on a server with two Intel ${ }^{\circledR}$ Xeon ${ }^{\circledR}$ X5650 processors at $2.66 \mathrm{GHz}$ and $49.4 \mathrm{GiB}$ of RAM

${ }^{7}$ As an indicator, direct-LS needs $849 \mathrm{MiB}$ of RAM to store $\boldsymbol{\Phi}$ in case (a) and $8.49 \mathrm{GiB}$ in case (b), whereas iter-LS or phase-LS need only $335 \mathrm{MiB}$ in both cases to store all $\boldsymbol{\Phi}_{\boldsymbol{n}, \boldsymbol{q}}$.

${ }^{8} 0.03 \mathrm{~s}$ in case (a) and $0.1 \mathrm{~s}$ in case (b).
} 


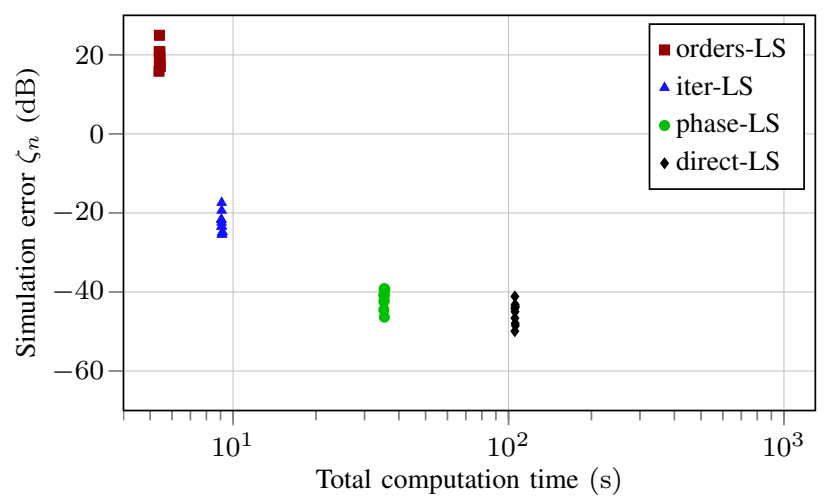

(a) $K=K_{1}=19$

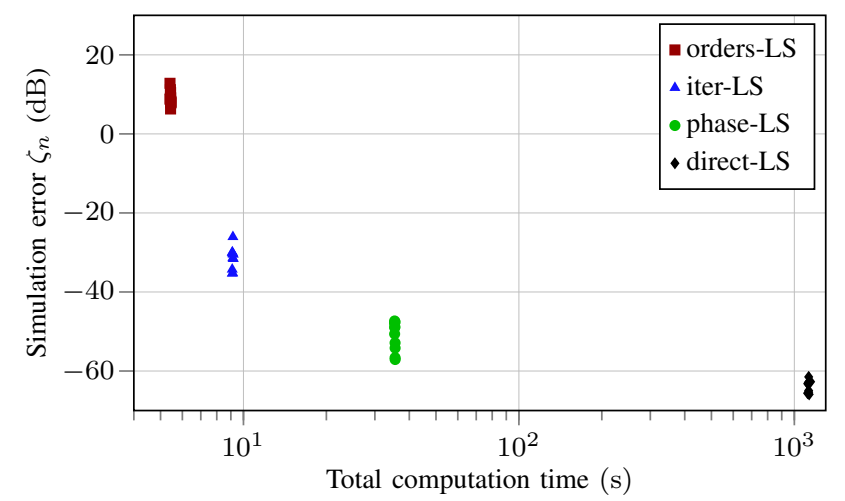

(b) $K=K_{2}=190$

Fig. 5. Comparison of simulation error $\varepsilon_{n}$ (in dB) w.r.t. the total computing time (in seconds) for the two number of tests signals $K$; each point represent the result of one experiment.

usage and computation time. Furthermore, this improvement has been compared with the one brought by a nonlinear order separation method relying on amplitude.

Future work will focus on the application of this prior separation stage to other type of identification methods, such as Cross-Correlation, or to specific structured systems, such as block-structured or Polynomial Nonlinear State-Space systems.

\section{REFERENCES}

[1] N. Wiener, "Response of a nonlinear device to noise," Massachusetts Inst. Technology, Rept, vol. 129, 1942.

[2] N. Wiener and T. Teichmann, "Nonlinear problems in random theory," Physics Today, vol. 12, p. 52, 1959.

[3] Y. Goussard, W. Krenz, and L. Stark, "An improvement of the Lee and Schetzen cross-correlation method," IEEE transactions on automatic control, vol. 30, no. 9, pp. 895-898, 1985.

[4] A. S. French and E. G. Butz, "Measuring the Wiener kernels of a nonlinear system using the fast Fourier transform algorithm," International Journal of Control, vol. 17, no. 3, pp. 529-539, 1973.

[5] M. Schetzen, "Measurement of the kernels of a non-linear system of finite order," International Journal of Control, vol. 1, no. 3, pp. 251263, 1965

[6] S. Clancy and W. Rugh, "A note on the identification of discretetime polynomial systems," IEEE Transactions on Automatic Control, vol. 24, no. 6, pp. 975-978, 1979.

[7] S. P. Boyd, Y. Tang, and L. O. Chua, "Measuring volterra kernels," Circuits and Systems, IEEE Transactions on, vol. 30, no. 8, pp. 571577, 1983.

[8] L. O. Chua and Y. Liao, "Measuring Volterra kernels (ii)," International journal of circuit theory and applications, vol. 17, no. 2, pp. 151-190, 1989.

[9] _ - "Measuring Volterra kernels (iii): How to estimate the highest significant order," International journal of circuit theory and applications, vol. 19, no. 2, pp. 189-209, 1991.

[10] M. J. Korenberg, "Functional expansions, parallel cascades and nonlinear difference equations," Advanced methods of physiological system modeling, vol. 1, pp. 221-240, 1987.

[11] Y. Goussard, W. C. Krenz, L. Stark, and G. Demoment, "Practical identification of functional expansions of nonlinear systems submitted to non-Gaussian inputs," Annals of biomedical engineering, vol. 19, no. 4, pp. 401-427, 1991.

[12] C. Cheng, Z. Peng, W. Zhang, and G. Meng, "Volterra-series-based nonlinear system modeling and its engineering applications: A stateof-the-art review," Mechanical Systems and Signal Processing, vol. 87, pp. 340-364, 2017.

[13] G. Favier, A. Y. Kibangou, and T. Bouilloc, "Nonlinear system modeling and identification using Volterra-PARAFAC models," International Journal of Adaptive Control and Signal Processing, vol. 26, no. 1, pp. 30-53, 2012.
[14] Y. Fu and G. Dumont, "An optimum time scale for discrete Laguerre network," IEEE Transactions on Automatic Control, vol. 38, no. 6, pp. 934-938, 1993.

[15] A. da Rosa, R. J. Campello, and W. C. Amaral, "Choice of free parameters in expansions of discrete-time Volterra models using Kautz functions," Automatica, vol. 43, no. 6, pp. 1084-1091, 2007.

[16] — - "Exact search directions for optimization of linear and nonlinear models based on generalized orthonormal functions," Department of Computer Sciences, University of São Paulo, Tech. Rep., 2008

[17] M. Rébillat, R. Hennequin, E. Corteel, and B. F. G. Katz, "Identification of cascade of Hammerstein models for the description of nonlinearities in vibrating devices," Journal of Sound and Vibration, vol. 330, no. 5, pp. 1018-1038, 2011.

[18] A. Y. Kibangou and G. Favier, "Identification of parallel-cascade Wiener systems using joint diagonalization of third-order Volterra kernel slices," Signal Processing Letters, IEEE, vol. 16, no. 3, pp. 188-191, 2009.

[19] — - "Toeplitz-Vandermonde Matrix Factorization With Application to Parameter Estimation of Wiener-Hammerstein Systems," Signal Processing Letters, IEEE, vol. 14, no. 2, pp. 141-144, 2007.

[20] D. Bouvier, T. Hélie, and D. Roze, "Nonlinear Homogeneous Order Separation for Volterra Series Identification," in Proceedings of the 20th International Conference on Digital Audio Effects (DAFx'17), Edinburgh, UK, 2017, pp. 3-10.

[21] W. J. Rugh, Nonlinear system theory. Johns Hopkins University Press Baltimore, 1981.

[22] S. P. Boyd, L. O. Chua, and C. A. Desoer, "Analytical foundations of Volterra series," IMA Journal of Mathematical Control and Information, vol. 1, no. 3, pp. 243-282, 1984.

[23] S. Boyd and L. Chua, "Fading memory and the problem of approximating nonlinear operators with Volterra series," IEEE Transactions on circuits and systems, vol. 32, no. 11, pp. 1150-1161, 1985.

[24] R. H. Lambert, "Vandermonde Method for Separation of Nonlinear Orders and Measurement of Linear Response," in Audio Engineering Society Convention 141. Audio Engineering Society, 2016.

[25] D. Bard and G. Sandberg, "Modeling of Nonlinearities in Electrodynamic Loudspeakers," in Audio Engineering Society Convention 123 Audio Engineering Society, 2007.

[26] B. Zhang and S. Billings, "Volterra series truncation and kernel estimation of nonlinear systems in the frequency domain," Mechanical Systems and Signal Processing, vol. 84, pp. 39-57, 2017.

[27] G. H. Golub and C. F. Van Loan, Matrix computations. Johns Hopkins University Press, 2012, vol. 3.

[28] P. J. Schreier and L. L. Scharf, Statistical signal processing of complex-valued data: the theory of improper and noncircular signals. Cambridge University Press, 2010.

[29] M. J. Korenberg, "Identifying nonlinear difference equation and functional expansion representations: the fast orthogonal algorithm," Annals of biomedical engineering, vol. 16, no. 1, pp. 123-142, 1988

[30] M. J. Korenberg, S. B. Bruder, and P. J. Mcllroy, "Exact orthogonal kernel estimation from finite data records: Extending Weiner's identification of nonlinear systems," Annals of biomedical engineering, vol. 16 , no. 2, pp. 201-214, 1988. 